\title{
Gastric Helicobacter pylori infection perturbs human oral microbiota
}

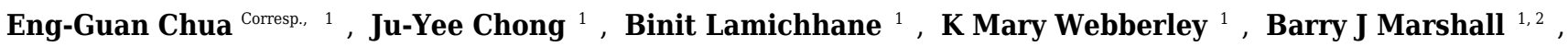 \\ Michael J Wise ${ }^{1,3}$, Chin-Yen Tay ${ }^{1,2}$ \\ ${ }^{1}$ The Marshall Centre for Infectious Diseases Research and Training, University of Western Australia, Crawley, Western Australia, Australia \\ 2 Shenzhen Dapeng New District Kuichong People Hospital, Shenzhen City, Guangdong Province, China \\ 3 Computer Science and Software Engineering, University of Western Australia, Crawley, Western Australia, Australia \\ Corresponding Author: Eng-Guan Chua \\ Email address: eng.chua@uwa.edu.au
}

\section{Background}

We investigated the effects of gastric Helicobacter pylori infection on the daytime and overnight human oral microbiota.

\section{Methods}

Twenty four volunteers were recruited. Ten tested positive for $H$. pylori infection by the Carbon-14 Urea Breath Test, and the rest were negative. Two oral swabs were collected: one immediately after waking up in the morning and before brushing teeth, and another in the evening before teeth-brushing. DNA extract acquired from each swab was subjected to Illumina sequencing of $16 S$ rRNA gene amplicons. The microbial abundance and composition were analysed in relation to $H$. pylori infection status.

\section{Results}

Helicobacter pylori-positive individuals had significant changes in the alpha and beta-diversities in the daytime samples in comparison to those who were $H$. pylori negative. To identify which taxa could be significantly affected within the cohorts in the daytime, we employed the LEfSe method. When compared against UBT-negative samples, significantly higher abundances were detected in both Pseudomonas and Roseomonas, while Fusobacterium, Solobacterium, Haemophilus and Streptococcus were significantly decreased in the UBT-positive samples.

\section{Discussion}

Our data demonstrated that $H$. pylori infection affects the human daytime oral microbiota. The hitherto undocumented changes of several bacterial genera due to $H$. pylori infection require more studies to examine their potential health effects on affected individuals. 


\section{Gastric Helicobacter pylori infection perturbs human oral microbiota}

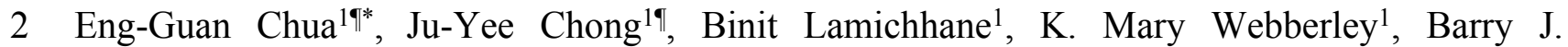

3 Marshall ${ }^{1,2}$, Michael J. Wise ${ }^{1,3}$, Chin-Yen Tay ${ }^{1,2}$

$4{ }^{1}$ The Marshall Centre for Infectious Diseases Research and Training, University of Western

5 Australia, Crawley, Western Australia, Australia

$6 \quad{ }^{2}$ Shenzhen Dapeng New District Kuichong People Hospital, Shenzhen City 518119, Guangdong

7 Province, China

$8{ }^{3}$ Computer Science and Software Engineering, University of Western Australia, Crawley 6009,

9 Western Australia, Australia

$11{ }^{*}$ Corresponding author

12 Eng-Guan Chua*

13 L Block, QEII Medical Centre, Monash Avenue, Crawley 6009, Western Australia, Australia

14 Email: eng.chua@uwa.edu.au

15

16 IEGC and JYC are Joint First Authors. 


\section{Abstract}

\section{Background}

19 We investigated the effects of gastric Helicobacter pylori infection on the daytime and overnight

20 human oral microbiota.

\section{Methods}

23 Twenty four volunteers were recruited. Ten tested positive for $H$. pylori infection by the Carbon-

2414 Urea Breath Test, and the rest were negative. Two oral swabs were collected: one 25 immediately after waking up in the morning and before brushing teeth, and another in the 26 evening before teeth-brushing. DNA extract acquired from each swab was subjected to Illumina 27 sequencing of $16 S r R N A$ gene amplicons. The microbial abundance and composition were 28 analysed in relation to $H$. pylori infection status.

\section{Results}

31 Helicobacter pylori-positive individuals had significant changes in the alpha and beta-diversities

32 in the daytime samples in comparison to those who were $H$. pylori negative. To identify which 33 taxa could be significantly affected within the cohorts in the daytime, we employed the LEfSe 34 method. When compared against UBT-negative samples, significantly higher abundances were 35 detected in both Pseudomonas and Roseomonas, while Fusobacterium, Solobacterium, 36 Haemophilus and Streptococcus were significantly decreased in the UBT-positive samples.

\section{Discussion}


39 Our data demonstrated that $H$. pylori infection affects the human daytime oral microbiota. The

40 hitherto undocumented changes of several bacterial genera due to $H$. pylori infection require

41 more studies to examine their potential health effects on affected individuals. 


\section{Introduction}

43 Helicobacter pylori is a highly adapted gastric pathogen that infects nearly half of the world's

44 population. It is an important etiological factor for the development of peptic and duodenal

45 ulcerations, chronic gastritis and gastric atrophy. In more severe, but less common cases, $H$.

46 pylori infection causes gastric adenocarcinoma and gastric mucosa-associated lymphoid tissue

47 (MALT) lymphoma (Marshall \& Windsor, 2005).

Although $H$. pylori is a well-known human gastric pathogen, it is less commonly regarded as a constituent of the oral cavity flora. However, it is listed in the Human Oral Microbiome Database

51 (Chen et al., 2010). Multiple previous PCR studies have detected H. pylori based on $16 S \mathrm{rRNA}$, $\operatorname{cag} A$ and $\operatorname{glm} M$ gene sequences in the saliva and dental plaque samples (Amiri et al., 2015;

53 Castro-Munoz et al., 2017; Silva et al., 2009). More importantly, several groups have reported

54 the presence of $H$. pylori in the oral cavity by culture, indicating that the human oral cavity can 55 act as a reservoir for $H$. pylori and therefore a potential source for transmission (Agarwal \& 56 Jithendra, 2012; Goosen et al., 2002; Teoman et al., 2007; Wang et al., 2014).

58 Following the lead of the earlier studies, we set out to detect $H$. pylori in the oral cavity. We used $16 \mathrm{~S}$ metagenomic sequencing to examine the presence of this bacterium in the oral cavities of 60 individuals who had their gastric infection status ascertained by the Urea Breath Test. We

61 hypothesised that the prevalence or abundance of $H$. pylori may vary with the time of day and so

62 took samples at two time points: in the morning before tooth brushing and in the evening. It is

63 thought that $H$. pylori might be predominantly deposited in the mouth by reflux overnight while

64 hosts were lying down, but that it may be removed by tooth brushing. We further hypothesised 
65 that gastric and/or oral H. pylori infection may lead to wider changes in the human oral

66 microbiome.

67

68 By testing at two times of day, we were also able to study the effect of $H$. pylori on what we

69 thought might be two different microbial groups. We expected the early morning sampling to

70 reveal the relationship between $H$. pylori infection and the predominantly anaerobic or

71 facultative anaerobic bacteria present in the oral cavity. We believed that during night time,

72 when one is asleep and is breathing normally via the nasal route, the oral cavity would remain

73 relatively closed for a prolonged period, generating a microaerobic or anaerobic condition that

74 favours the growth of anaerobes or facultative anaerobes (Fitzpatrick et al., 2003). The other

75 samples were taken in the evening when the oral cavity is anticipated to be in an aerobic state

76 due to the mouth opening for activities such as eating and drinking, and talking. This design

77 allowed us to investigate the potential effect of $H$. pylori infection to influence the structure of

78 both the anaerobic and aerobic bacterial communities. These results would enable us to further

79 assess the changes, if any, in oral microbiome on the health of human host.

80

\section{Materials and methods}

82 Ethics approval and consent to participate

83 This study was approved by the Human Ethics of the University of Western Australia

84 (RA/4/1/7953). Written consent was obtained from all individuals participated in this study.

85

\section{Sample collection}


87 A total of 24 University of Western Australia postgraduate students who brushed their teeth 88 twice a day (after waking up and before going to bed) were recruited over a 6-month period and 89 screened for H. pylori infection using the PYtest Urea Breath Test (UBT) kit (Tri-Med, Western 90 Australia, Australia). Two oral swabs, rotated along the inside of the cheek for approximately 10 91 seconds, were collected by study participants: one in the morning immediately after waking up 92 and another at six in the evening, both before teeth-brushing. The morning samples allowed us 93 to characterise the oral microbial community composition of the post overnight cycle whilst the 94 latter focused on the daytime oral microbiome. For consistency, the morning and evening 95 samples are referred to as the overnight and daytime samples, respectively.

$97 \quad 16 \mathrm{~S}$ amplicons generation and sequencing

98 Oral swab DNA extraction was performed using DNeasy ${ }^{\circledR}$ blood and tissue kit (Qiagen, Hilden, 99 Germany). The V3-V4 region of the 16S rRNA gene was amplified from each DNA extract 100 using the S-D-Bact-0341-b-S-17 (5'-CCTACGGGNGGCWGCAG-3') and S-D-Bact-0785-a-A10121 (5'-GACTACHVGGGTATCTAATCC-3') primer pair (Klindworth et al., 2013). Illumina 102 adapter overhang sequences, 5'-TCGTCGGCAGCGTCAGATGTGTATAAGAGACAG-3' and 103 5'-GTCTCGTGGGCTCGGAGATGTGTATAAGAGACAG-3', were added to the 5' ends of

104 the forward and reverse primers, respectively. Initial PCR amplification of 16s RNA genes was 105 performed using Taq DNA polymerase (New England Biolabs, Ipswich, United States) with the 106 following conditions: an initial denaturation at $95^{\circ} \mathrm{C}$ for 30 seconds, followed by 30 cycles 107 consisting of denaturation at $95^{\circ} \mathrm{C}$ for 10 seconds, annealing at $55^{\circ} \mathrm{C}$ for 30 seconds and 108 extension at $72^{\circ} \mathrm{C}$ for 30 seconds, and a final extension step at $72^{\circ} \mathrm{C}$ for 5 minutes. A PCR clean109 up was carried out using Agencourt Ampure XP beads (Beckman Coulter, Brea, United States) 
110 prior to performing index PCR using Nextera XT index kit (Illumina, San Diego, United States).

111 The amplicons were sequenced using the $2 \times 250$ paired-end protocol (MiSeq Reagent Kit v2 for

112500 cycles) on an Illumina MiSeq instrument.

\section{3}

\section{Data analysis}

115 The MiSeq-generated sequence data were trimmed via Sickle (github.com/najoshi/sickle) using

116 the following parameters: -q $20-1$ 200. Merging of paired-end sequences, filtering of chimeric

117 sequences, clustering of 16S rRNA sequences into Operational Taxonomic Units (OTUs) and

118 taxonomic classification of OTU were conducted using Micca version 1.6.2 (Albanese et al.,

119 2015). Briefly, micca mergepairs was run to merge paired-end sequences with a minimum

120 overlap length of $25 \mathrm{bp}$ and maximum allowed mismatches of 5 . The output sequences were then

121 subjected to micca filter to retain sequences that have a minimum length of $400 \mathrm{bp}$ and an

122 expected error rate of not greater than $0.5 \%$. Subsequently micca otu was executed to allow

123 filtering of chimeric sequences and de novo clustering of sequences into OTUs with a 97\%

124 identity threshold.

125

126 Taxonomic assignment of the de novo-clustered OTUs was performed using the Bayesian LCA-

127 based taxonomic classification method with a 1e-100 cut-off e-value and 100 bootstrap

128 replications, against the NCBI 16S microbial database (Coordinators, 2018; Gao et al., 2017).

129 Taxonomic assignment at each level was accepted only with a minimum confidence score of 80.

130 Multiple sequence alignment of the OTU representative sequences was performed using PASTA

131 (Mirarab et al., 2015). A phylogenetic tree was constructed using FastTree under the GTR+CAT

132 model (Price et al., 2010). 
134 The rarefaction depth values were set at 5249 and 3305, respectively, for the overnight and

135 daytime samples, prior to further analysis using QIIME (version 1.9.1) to compare alpha and

136 beta diversities between UBT-positive and UBT-negative individuals (Caporaso et al., 2010).

137 Alpha diversity was evaluated based on the following metrics: observed species, Shannon index

138 and Chaol index. Non-parametric two-sample $t$-test with Bonferroni correction was used to

139 compare the alpha diversity metrics between groups. Principle Coordinates Analysis (PCoA)

140 using weighted UniFrac distance metric was performed to visualize separation of samples. Non-

141 parametric statistical analysis of the distance metric was performed using ANOSIM with 1000

142 permutations.

143

144 Statistical analysis

145 LEfSe was employed to identify distinguishing bacterial phyla and genera between the $H$. pylori-

146 positive and negative cohorts (Segata et al., 2011).

147

\section{Accession numbers}

149 All sequencing data generated in this study have been submitted to Sequence Read Archives (SRA)

150 database with their accession numbers listed in Table S1.

151

\section{Results}

153 Among the 24 participants who underwent the Carbon-14 Urea Breath Test, 10 and 14 were

154 determined as $H$. pylori positive and negative, respectively. The demographic characteristics of 155 the participants are summarised in Table 1. 


\section{$157 \quad 16 S$ rRNA gene sequencing}

158 A total of 5,561,542 and 8,258,347 reads were generated for the overnight and daytime samples,

159 respectively. Following quality trimming, merging and filtering of paired-end sequences,

160628,351 with an average read length of $461.5 \pm 7.5 \mathrm{bp}$ were retained for the overnight samples.

161 For the daytime samples, 906,743 reads with an average length of $461.8 \pm 7.3$ bp were obtained.

162 These reads were further clustered into 146 and 137 OTUs for the overnight and daytime 163 datasets, respectively, after the removal of chimeric sequences.

\section{Characterization of the oral microbiota}

166 The bacterial communities in the UBT-negative and UBT-positive cohorts of both periods were 167 analysed at phylum and genus levels (Tables S2 and S3, respectively). During the day,

168 Firmicutes, Proteobacteria and Actinobacteria constituted the top three most abundant phyla, 169 accounting for $43.9 \%, 34.5 \%$ and $17.7 \%$ of the oral microbiota, respectively. In the overnight 170 samples, the top three most abundant phyla were Firmicutes, Proteobacteria and Bacteroidetes, 171 with relative abundances of $51.9 \%, 29.2 \%$ and $10.8 \%$, respectively. At the genus level and by 172 using a minimum frequency threshold of 90\%, Rothia and Streptococcus were identified in most 173 samples of both time periods, with daytime relative abundances of $14.2 \%$ and $30.4 \%$, and $5 \%$ 174 and $42.6 \%$ in overnight, respectively (Fig. 1). In addition to the two genera mentioned above, 175 Actinomyces, Gemella, Granulicatella, Haemophilus and Veillonella were shared between most 176 overnight samples, together representing $16.2 \%$ of the oral microbiota. Notably, we were unable 177 to detect any Helicobacter sequences from our samples in this study. 


\section{Diversity and LEfSe analyses}

180 To estimate the alpha diversity of the oral microbial community, we employed observed species,

181 Shannon diversity and Chaol richness estimator indexes. As depicted in Figure 2, the rarefaction

182 curves of Shannon diversity index for all daytime and overnight plateaued, indicating that there

183 is sufficient coverage of microbial diversity per sample. Further comparisons between the UBT-

184 positive and UBT-negative cohorts by the Shannon diversity index demonstrated that during the

185 day, the former exhibits significantly reduced oral microbial diversity compared with that of the

186 latter $(p$-value $=0.03)($ Fig. 3). At night, however, no significant difference in microbial diversity

187 could be observed.

188

189 Principal Coordinates Analysis (PCoA) based on weighted UniFrac index was performed at the

190 OTU level to assess if there is any grouping of the samples by sharing similar bacterial

191 communities. No obvious separation was observed in the overnight samples (Fig. 4). In the

192 daytime samples, however, we observed a good clustering of the bacterial communities in the

193 UBT-negative cohort (Fig. 5). The UBT-positive samples displayed an irregular distribution

194 pattern, suggesting that $H$. pylori infection disturbs the oral microbiome during the day. An

195 analysis of similarities (ANOSIM) non-parametric statistical test based on weighted UniFrac

196 distance further revealed that there is a significant difference in the bacterial community

197 composition between the cohorts during the day ( $p$-value $=0.003, R=0.343$ ).

198

199 To identify which taxa could be significantly affected within the cohorts in the daytime, we

200 employed the linear discriminant analysis effect size (LEfSe) method. At the phylum level,

201 Proteobacteria were significantly enriched in UBT-positive samples whilst Fusobacteria and 
202 Firmicutes were significantly reduced (Fig. 6A). At the genus level, in UBT-positive samples,

203 significantly higher abundances were detected in both Pseudomonas and Roseomonas, while

204 Fusobacterium, Solobacterium, Haemophilus and Streptococcus were significantly decreased

205 (Fig. 6B). We attempted the analysis on the overnight samples, but identified no distinguishing

206 taxa, in agreement with the PCoA outcome which also showed no obvious separation between

207 cohorts.

208

209 Discussion

210 In this study, the effect of $H$. pylori infection on the oral microbiota was explored. In our

211 sequencing data, no trace of Helicobacter $16 S r R N A$ gene sequence could be detected at either

212 time point among the $10 \mathrm{H}$. pylori-positive volunteers recruited in this study. We also attempted

213 PCR detection using $H$. pylori-specific primers targeting $16 S$ rRNA, ureI and vacA genes.

214 However, these yielded no positive outcomes (data not shown). In a recent meta-study, it was

215 demonstrated that the presence of $H$. pylori in saliva detected via either PCR or culture methods

216 was less than that from dental plaque (Anand et al., 2014). Hence the absence of Helicobacter

217 sequence in our study could be attributed to our oral swab collection method that did not include

218 any dental plaque material, or it may be that $H$. pylori was genuinely absent in the oral cavity of

219 these UBT-positive individuals on the days covered by the samples.

220

221 The core bacterial genera identified in our study were in agreement with the findings elsewhere

222 reported (Bik et al., 2010; Seoudi et al., 2015). Actinomyces, Gemella and Veillonella were more

223 prevalent in the overnight samples, consistent with their anaerobic characteristic (Delwiche et al.,

224 1985; Valour et al., 2014; Vasishtha et al., 1996). The remaining genera including Rothia, 
225 Streptococcus, Granulicatella and Haemophilus have been shown to contain most, if not all,

226 species that are facultative anaerobes (Collins \& Lawson, 2000; Kuklinska \& Kilian, 1984;

227 Tunney et al., 2008).

228

229 Despite the fact that the presence of Helicobacter genus could not be detected in any of our 230 samples, significant differences in both alpha and beta diversities were observed in H. pylori231 positive individuals relative to the negative comparison group during the day. It has been shown

232 that $H$. pylori infection is associated with the systemic changes of different metabolic and 233 immunological factors, so it is possible that any of these changes would perturb the stability of 234 the oral microbial community (Buzas, 2014). It is also interesting to observe that such changes 235 targeted only the daytime oral microbiota but not those at night. There has been abundant 236 evidence that $H$. pylori is capable of colonising the oral cavity and since H. pylori is an obligate 237 aerobe, a possible explanation would be that during the day, the proliferation of $H$. pylori 238 changes the nutrient availability and possibly $\mathrm{pH}$ within the oral cavity, in addition to the 239 secretion of $H$. pylori proteins and metabolites (Anand et al., 2014). These possible events, 240 together, may affect the growth of certain bacterial species and thus altering the structure of the 241 daytime oral microbiota.

243 Further LEfSe analysis at the genus level revealed six genera that were different between both

244 UBT-positive and UBT-negative cohorts in the daytime. Among the four genera that exhibited 245 remarkably lower abundances in the UBT-positive samples, Fusobacterium and Solobacterium 246 have been implicated in periodontal disease (Schirrmeister et al., 2009; Signat et al., 2011).

247 Streptococcus, on the hand, contains oral members that are opportunistic pathogens commonly 
248 associated with the development of cardiovascular diseases (Adam et al., 2015; Nakano et al., 249 2009). It is noteworthy that both Fusobacterium and Solobacterium are often associated, and that

250 Fusobacterium and Streptococcus could form aggregates with each other, reflecting a potentially

251 synergistic and/or symbiotic relationship between them that may enhance bacterial survival

252 fitness for long term colonisation of the human oral cavity (Edwards et al., 2006; Kaplan et al., 253 2009; Schirrmeister et al., 2009). Meanwhile, albeit statistically significant, the clinical 254 importance of Solobacterium should be carefully examined owing to its low sequence counts 255 among the UBT-negative samples. Of the significantly diminished Haemophilus bacterial 256 population in our UBT-positive samples, four species were identified including H. haemolyticus, 257 H. paraphrohaemolyticus, $H$. pittmaniae and $H$. parainfluenzae, which are common bacterial 258 flora of the oral cavity (Kuklinska \& Kilian, 1984). While Haemophilus spp. are generally 259 regarded as harmless to human host, $H$. parainfleunzae has been reported as an opportunistic 260 pathogen for causing respiratory tract infections and endocarditis (Darras-Joly et al., 1997; Rhind 261 et al., 1985). The apparent inhibitory effect of $H$. pylori infection on the growth on these 262 opportunistic pathogens in our study, but only in the daytime, is intriguing. It is uncertain 263 whether such inhibition would reduce the risk of developing diseases associated with these 264 bacteria.

266 In this study, Pseudomonas and Roseomonas were significantly enriched in the UBT-positive 267 samples. While members of the genus Pseudomonas rarely cause disease in healthy individuals 268 and only $P$. aeruginosa has been widely reported on its role in respiratory tract infection in 269 immunocompromised patients, the latter was not detected in our UBT-positive samples (Rivas 270 Caldas \& Boisrame, 2015). It is noteworthy that no Pseudomonas sequence was present in any of 
271 the UBT-negative samples. Although the Pseudomonas species identified in this study had no

272 identified disease correlations, their presence in the oral cavity of H. pylori infected individuals,

273 and thus the potential impact on one's general health, should be carefully assessed. Similar to

274 Pseudomonas, human infections caused by Roseomonas species are uncommon (Wang et al.,

275 2012). The detection of Roseomonas only among the UBT-positives, but not in any UBT-

276 negatives, during the day is again interesting. Nevertheless, like Solobacterium, its clinical

277 relevance should be interpreted cautiously due to its very low abundance in $H$. pylori infected

278 individuals.

279

280 Conclusions

281 Our study reported the effects of $H$. pylori infection on the human oral microbiome, particularly 282 during the day. The abundance levels of several bacterial genera including opportunistic 283 pathogens were significantly affected by $H$. pylori infection. To better understand and validate 284 the effects of H. pylori infection on the human oral microbiota, another study entailing a greater 285 sample size would be necessary. Also, analysing the oral microbiota with the same person after 286 eradication would help to resolve whether $H$. pylori infection directly affects the oral 287 microbiome. Further studies may be required to elucidate the impacts of these bacterial 288 community changes on the general health of individuals with $H$. pylori infection.

\section{Acknowledgements}

291 We would like to thank Tri-Med Distributors Pty Ltd for providing the UBT screening service. 


\section{References}

293

294

295

296

297

298

299

300

301

302

303

304

305

306

307

308

309

310

311

312

313

314

315

316

317

318

319

320

321

322

323

324

325

326

327

328

329

330

331

332

333

334

335

Adam EL, Siciliano RF, Gualandro DM, Calderaro D, Issa VS, Rossi F, Caramelli B, Mansur AJ, and Strabelli TM. 2015. Case series of infective endocarditis caused by Granulicatella species. International journal of infectious diseases: IJID : official publication of the International Society for Infectious Diseases 31:56-58. 10.1016/j.ijid.2014.10.023

Agarwal S, and Jithendra KD. 2012. Presence of Helicobacter pylori in subgingival plaque of periodontitis patients with and without dyspepsia, detected by polymerase chain reaction and culture. Journal of Indian Society of Periodontology 16:398-403. 10.4103/0972124X.100919

Albanese D, Fontana P, De Filippo C, Cavalieri D, and Donati C. 2015. MICCA: a complete and accurate software for taxonomic profiling of metagenomic data. Sci Rep 5:9743. 10.1038/srep09743

Amiri N, Abiri R, Eyvazi M, Zolfaghari MR, and Alvandi A. 2015. The frequency of Helicobacter pylori in dental plaque is possibly underestimated. Archives of oral biology 60:782-788. 10.1016/j.archoralbio.2015.02.006

Anand PS, Kamath KP, and Anil S. 2014. Role of dental plaque, saliva and periodontal disease in Helicobacter pylori infection. World journal of gastroenterology 20:5639-5653. 10.3748/wjg.v20.i19.5639

Bik EM, Long CD, Armitage GC, Loomer P, Emerson J, Mongodin EF, Nelson KE, Gill SR, Fraser-Liggett CM, and Relman DA. 2010. Bacterial diversity in the oral cavity of 10 healthy individuals. The ISME journal 4:962-974. 10.1038/ismej.2010.30

Buzas GM. 2014. Metabolic consequences of Helicobacter pylori infection and eradication. World journal of gastroenterology 20:5226-5234. 10.3748/wjg.v20.i18.5226

Caporaso JG, Kuczynski J, Stombaugh J, Bittinger K, Bushman FD, Costello EK, Fierer N, Pena AG, Goodrich JK, Gordon JI, Huttley GA, Kelley ST, Knights D, Koenig JE, Ley RE, Lozupone CA, McDonald D, Muegge BD, Pirrung M, Reeder J, Sevinsky JR, Turnbaugh PJ, Walters WA, Widmann J, Yatsunenko T, Zaneveld J, and Knight R. 2010. QIIME allows analysis of high-throughput community sequencing data. Nature Methods 7:335336. 10.1038/nmeth.f.303

Castro-Munoz LJ, Gonzalez-Diaz CA, Munoz-Escobar A, Tovar-Ayona BJ, Aguilar-Anguiano LM, Vargas-Olmos R, and Sanchez-Monroy V. 2017. Prevalence of Helicobacter pylori from the oral cavity of Mexican asymptomatic children under 5 years of age through PCR. Archives of oral biology 73:55-59. 10.1016/j.archoralbio.2016.09.007

Chen T, Yu WH, Izard J, Baranova OV, Lakshmanan A, and Dewhirst FE. 2010. The Human Oral Microbiome Database: a web accessible resource for investigating oral microbe taxonomic and genomic information. Database (Oxford) 2010:baq013. 10.1093/database/baq013

Collins MD, and Lawson PA. 2000. The genus Abiotrophia (Kawamura et al.) is not monophyletic: proposal of Granulicatella gen. nov., Granulicatella adiacens comb. nov., Granulicatella elegans comb. nov. and Granulicatella balaenopterae comb. nov. International journal of systematic and evolutionary microbiology $50 \mathrm{Pt} \mathrm{1:365-369.}$ 10.1099/00207713-50-1-365

Coordinators NR. 2018. Database resources of the National Center for Biotechnology Information. Nucleic Acids Res 46:D8-D13. 10.1093/nar/gkx1095 
336

337

338

339

340

341

342

343

344

345

346

347

348

349

350

351

352

353

354

355

356

357

358

359

360

361

362

363

364

365

366

367

368

369

370

371

372

373

374

375

376

377

378

379

380

Darras-Joly C, Lortholary O, Mainardi JL, Etienne J, Guillevin L, and Acar J. 1997. Haemophilus endocarditis: report of 42 cases in adults and review. Haemophilus Endocarditis Study Group. Clin Infect Dis 24:1087-1094.

Delwiche EA, Pestka JJ, and Tortorello ML. 1985. The veillonellae: gram-negative cocci with a unique physiology. Annual review of microbiology 39:175-193. 10.1146/annurev.mi.39.100185.001135

Edwards AM, Grossman TJ, and Rudney JD. 2006. Fusobacterium nucleatum transports noninvasive Streptococcus cristatus into human epithelial cells. Infection and immunity 74:654-662. 10.1128/IAI.74.1.654-662.2006

Fitzpatrick MF, Driver HS, Chatha N, Voduc N, and Girard AM. 2003. Partitioning of inhaled ventilation between the nasal and oral routes during sleep in normal subjects. Journal of applied physiology 94:883-890. 10.1152/japplphysiol.00658.2002

Gao X, Lin H, Revanna K, and Dong Q. 2017. A Bayesian taxonomic classification method for $16 \mathrm{~S}$ rRNA gene sequences with improved species-level accuracy. BMC Bioinformatics 18:247. 10.1186/s12859-017-1670-4

Goosen C, Theron J, Ntsala M, Maree FF, Olckers A, Botha SJ, Lastovica AJ, and van der Merwe SW. 2002. Evaluation of a novel heminested PCR assay based on the phosphoglucosamine mutase gene for detection of Helicobacter pylori in saliva and dental plaque. Journal of clinical microbiology 40:205-209.

Kaplan CW, Lux R, Haake SK, and Shi W. 2009. The Fusobacterium nucleatum outer membrane protein RadD is an arginine-inhibitable adhesin required for inter-species adherence and the structured architecture of multispecies biofilm. Molecular microbiology 71:35-47. 10.1111/j.1365-2958.2008.06503.x

Klindworth A, Pruesse E, Schweer T, Peplies J, Quast C, Horn M, and Glockner FO. 2013. Evaluation of general 16S ribosomal RNA gene PCR primers for classical and nextgeneration sequencing-based diversity studies. Nucleic Acids Res 41:e1. 10.1093/nar/gks808

Kuklinska D, and Kilian M. 1984. Relative proportions of Haemophilus species in the throat of healthy children and adults. Eur J Clin Microbiol 3:249-252.

Marshall BJ, and Windsor HM. 2005. The relation of Helicobacter pylori to gastric adenocarcinoma and lymphoma: pathophysiology, epidemiology, screening, clinical presentation, treatment, and prevention. Med Clin North Am 89:313-344, viii. 10.1016/j.mcna.2004.09.001

Mirarab S, Nguyen N, Guo S, Wang LS, Kim J, and Warnow T. 2015. PASTA: Ultra-Large Multiple Sequence Alignment for Nucleotide and Amino-Acid Sequences. Journal of computational biology : a journal of computational molecular cell biology 22:377-386. $10.1089 / \mathrm{cmb} .2014 .0156$

Nakano K, Nemoto H, Nomura R, Inaba H, Yoshioka H, Taniguchi K, Amano A, and Ooshima T. 2009. Detection of oral bacteria in cardiovascular specimens. Oral microbiology and immunology 24:64-68. 10.1111/j.1399-302X.2008.00479.x

Price MN, Dehal PS, and Arkin AP. 2010. FastTree 2--approximately maximum-likelihood trees for large alignments. PLoS One 5:e9490. 10.1371/journal.pone.0009490

Rhind GB, Gould GA, Ahmad F, Croughan MJ, and Calder MA. 1985. Haemophilus parainfluenzae and $\mathrm{H}$ influenzae respiratory infections: comparison of clinical features. Br Med J (Clin Res Ed) 291:707-708. 
381

382

383

384

385

386

387

388

389

390

391

392

393

394

395

396

397

398

399

400

401

402

403

404

405

406

407

408

409

410

411

412

413

414

415

416

417

418

Rivas Caldas R, and Boisrame S. 2015. Upper aero-digestive contamination by Pseudomonas aeruginosa and implications in Cystic Fibrosis. J Cyst Fibros 14:6-15. 10.1016/j.jcf.2014.04.008

Schirrmeister JF, Liebenow AL, Pelz K, Wittmer A, Serr A, Hellwig E, and Al-Ahmad A. 2009. New bacterial compositions in root-filled teeth with periradicular lesions. $J$ Endod 35:169-174. 10.1016/j.joen.2008.10.024

Segata N, Izard J, Waldron L, Gevers D, Miropolsky L, Garrett WS, and Huttenhower C. 2011. Metagenomic biomarker discovery and explanation. Genome Biol 12:R60. 10.1186/gb2011-12-6-r60

Seoudi N, Bergmeier LA, Drobniewski F, Paster B, and Fortune F. 2015. The oral mucosal and salivary microbial community of Behcet's syndrome and recurrent aphthous stomatitis. Journal of oral microbiology 7:27150. 10.3402/jom.v7.27150

Signat B, Roques C, Poulet P, and Duffaut D. 2011. Fusobacterium nucleatum in periodontal health and disease. Curr Issues Mol Biol 13:25-36.

Silva DG, Stevens RH, Macedo JM, Albano RM, Falabella ME, Veerman EC, and Tinoco EM. 2009. Detection of cytotoxin genotypes of Helicobacter pylori in stomach, saliva and dental plaque. Archives of oral biology 54:684-688. 10.1016/j.archoralbio.2009.04.006

Teoman I, Ozmeric N, Ozcan G, Alaaddinoglu E, Dumlu S, Akyon Y, and Balos K. 2007. Comparison of different methods to detect Helicobacter pylori in the dental plaque of dyspeptic patients. Clinical oral investigations 11:201-205. 10.1007/s00784-007-0104-5

Tunney MM, Field TR, Moriarty TF, Patrick S, Doering G, Muhlebach MS, Wolfgang MC, Boucher R, Gilpin DF, McDowell A, and Elborn JS. 2008. Detection of anaerobic bacteria in high numbers in sputum from patients with cystic fibrosis. Am J Respir Crit Care Med 177:995-1001. 10.1164/rccm.200708-11510C

Valour F, Senechal A, Dupieux C, Karsenty J, Lustig S, Breton P, Gleizal A, Boussel L, Laurent F, Braun E, Chidiac C, Ader F, and Ferry T. 2014. Actinomycosis: etiology, clinical features, diagnosis, treatment, and management. Infect Drug Resist 7:183-197. 10.2147/IDR.S39601

Vasishtha S, Isenberg HD, and Sood SK. 1996. Gemella morbillorum as a cause of septic shock. Clin Infect Dis 22:1084-1086.

Wang CM, Lai CC, Tan CK, Huang YC, Chung KP, Lee MR, Hwang KP, and Hsueh PR. 2012. Clinical characteristics of infections caused by Roseomonas species and antimicrobial susceptibilities of the isolates. Diagn Microbiol Infect Dis 72:199-203. 10.1016/j.diagmicrobio.2011.11.013

Wang XM, Yee KC, Hazeki-Taylor N, Li J, Fu HY, Huang ML, and Zhang GY. 2014. Oral Helicobacter pylori, its relationship to successful eradication of gastric H. pylori and saliva culture confirmation. Journal of physiology and pharmacology : an official journal of the Polish Physiological Society 65:559-566. 


\section{Figure 1}

Core bacterial genera identified among the daytime and overnight samples.

A minimum threshold of 0.9 was employed for the identification of core genera among the daytime and overnight samples.

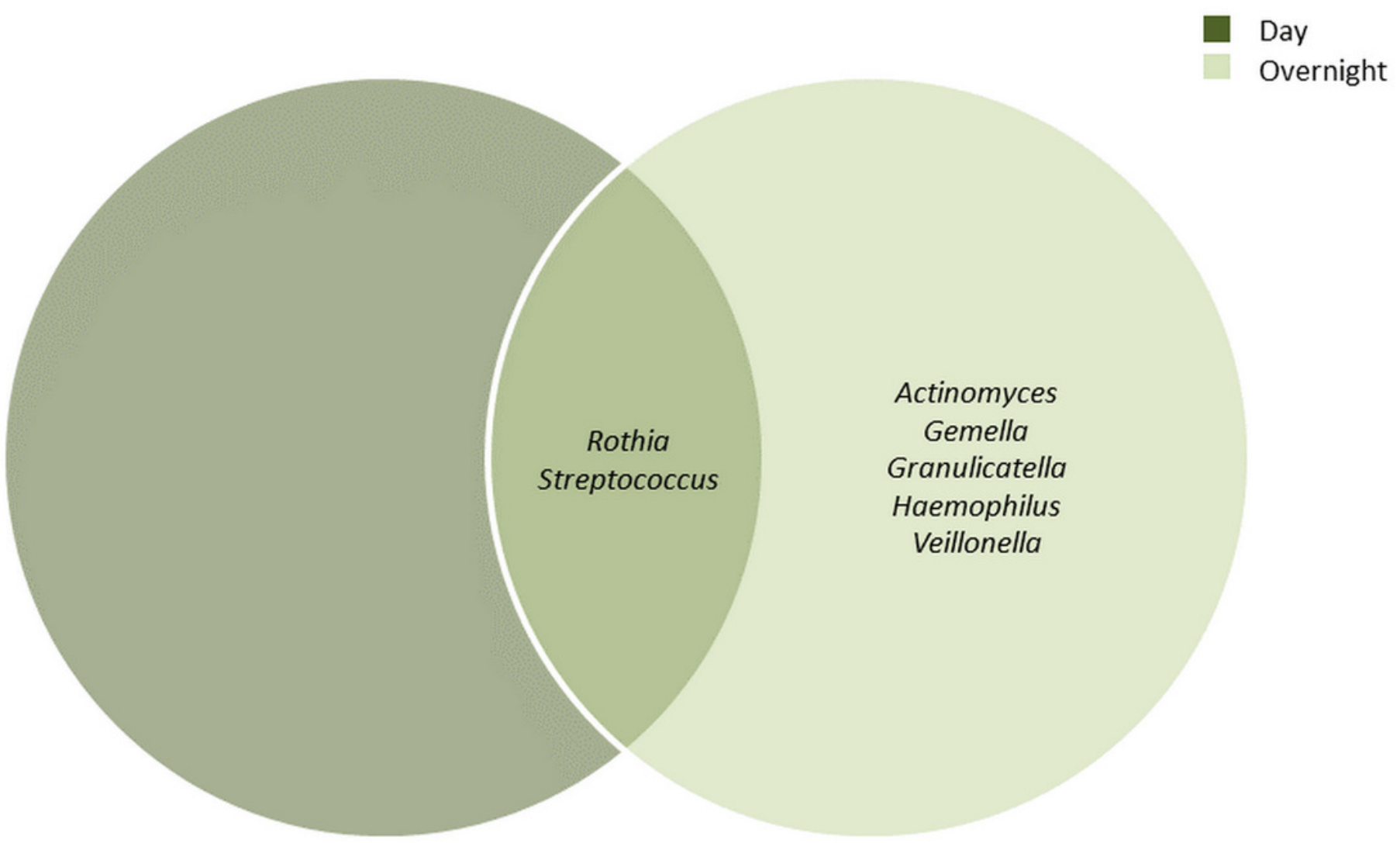


Figure 2

Rarefaction curve plots of the daytime and overnight samples.

(A) Rarefaction curve of daytime samples based on the numbers of observed species. (B) Rarefaction curve of overnight samples based on the numbers of observed species. (C) Rarefaction curve of daytime samples based on Shannon diversity index. (D) Rarefaction curve of overnight samples based on Shannon diversity index.
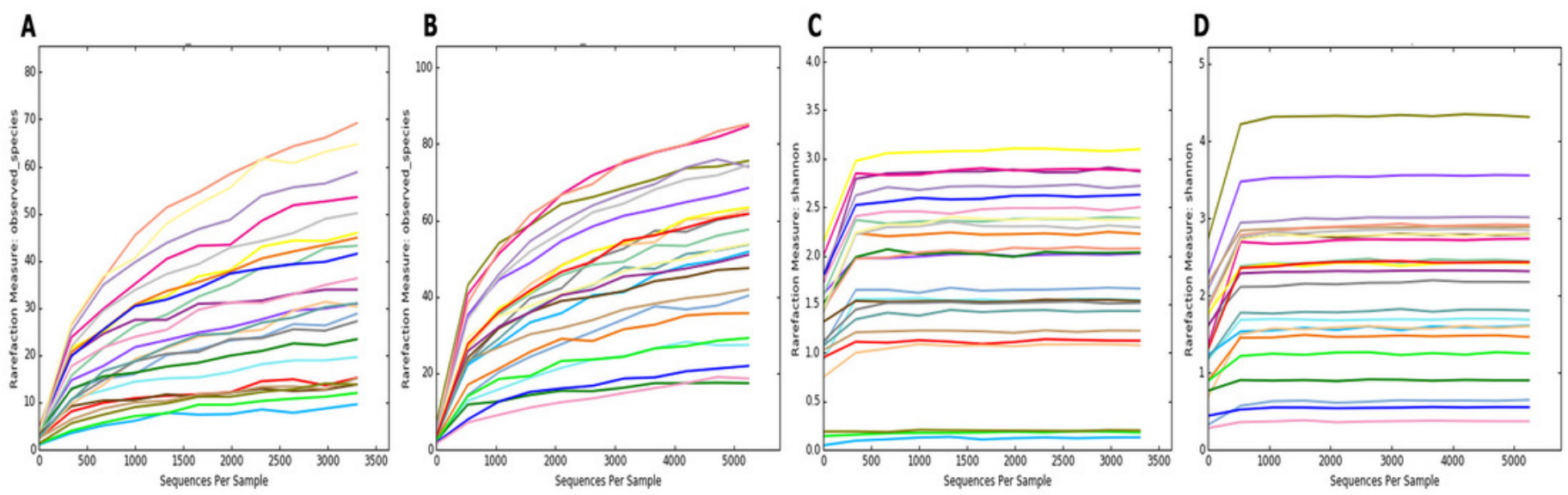
Figure 3

Daytime comparison of microbial diversity between the UBT-negative and UBT-positive cohorts.

Boxplot of Shannon index depicts significant differences in daytime bacterial diversity between the UBT-negative and UBT-postive cohorts.

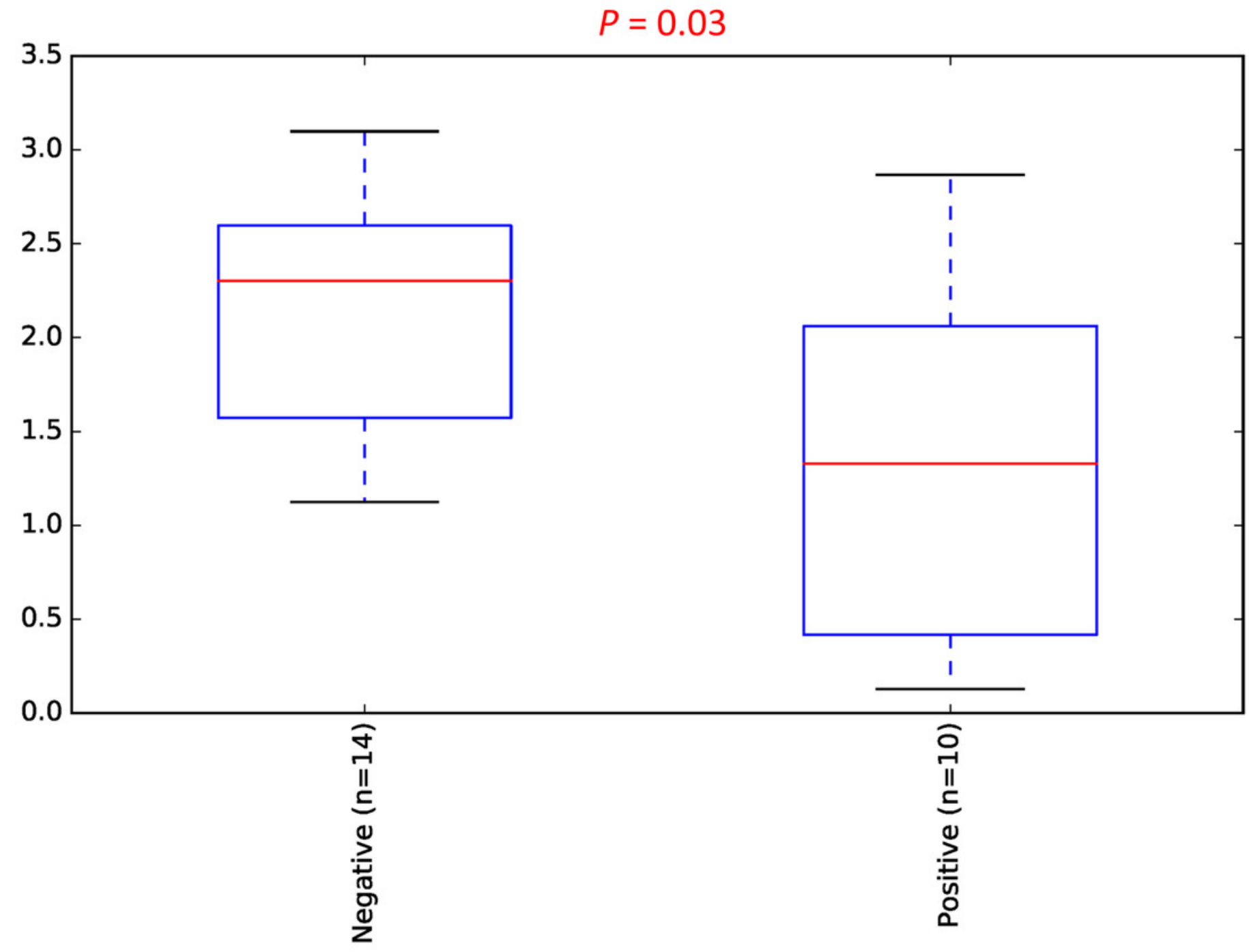


Figure 4

PCOA plot based on weighted UniFrac distance metric comparing overnight OTU abundances between UBT-positive and UBT-negative cohorts.

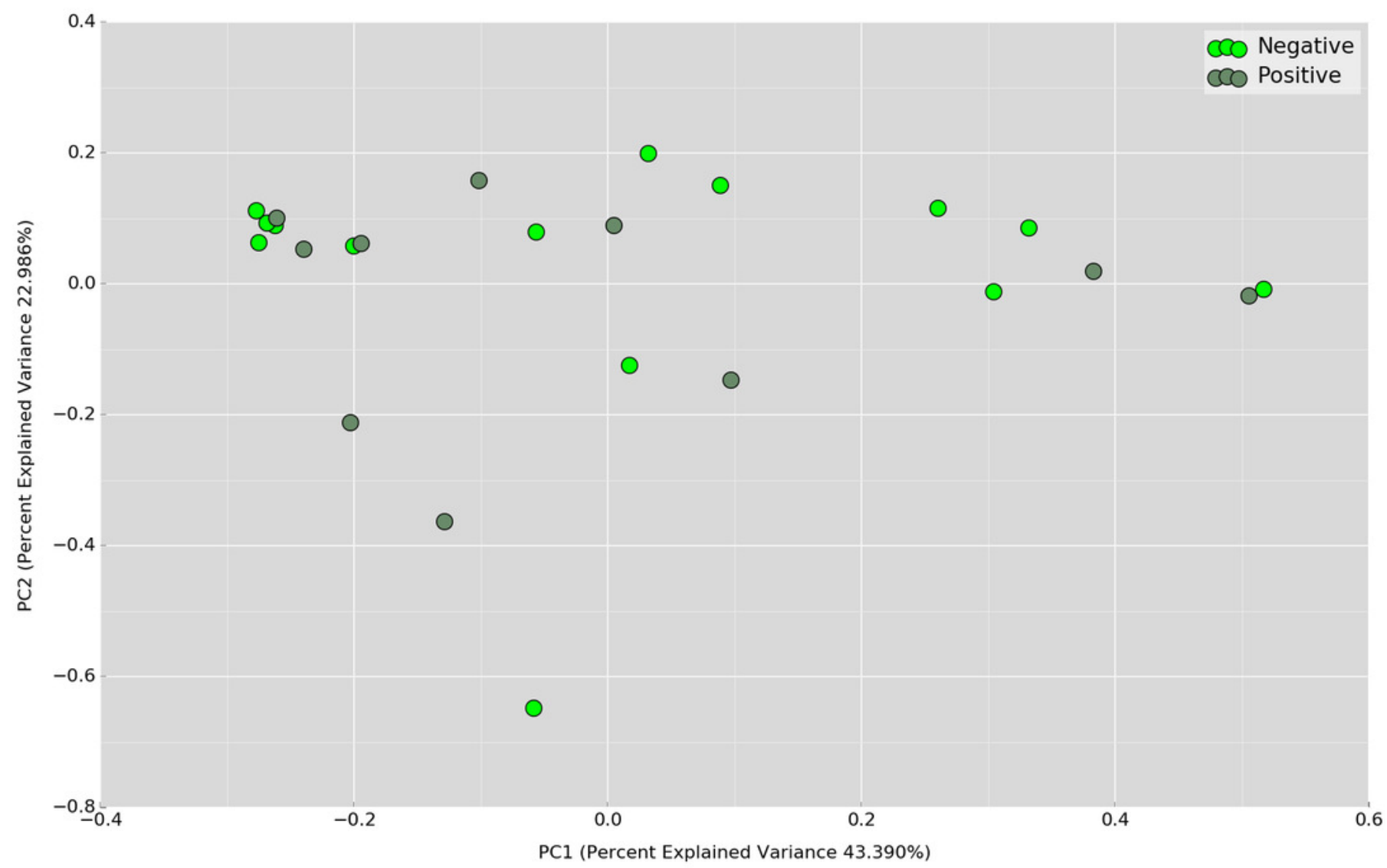


Figure 5

PCoA plot based on weighted UniFrac distance metric comparing daytime OTU abundances between UBT-positive and UBT-negative cohorts.

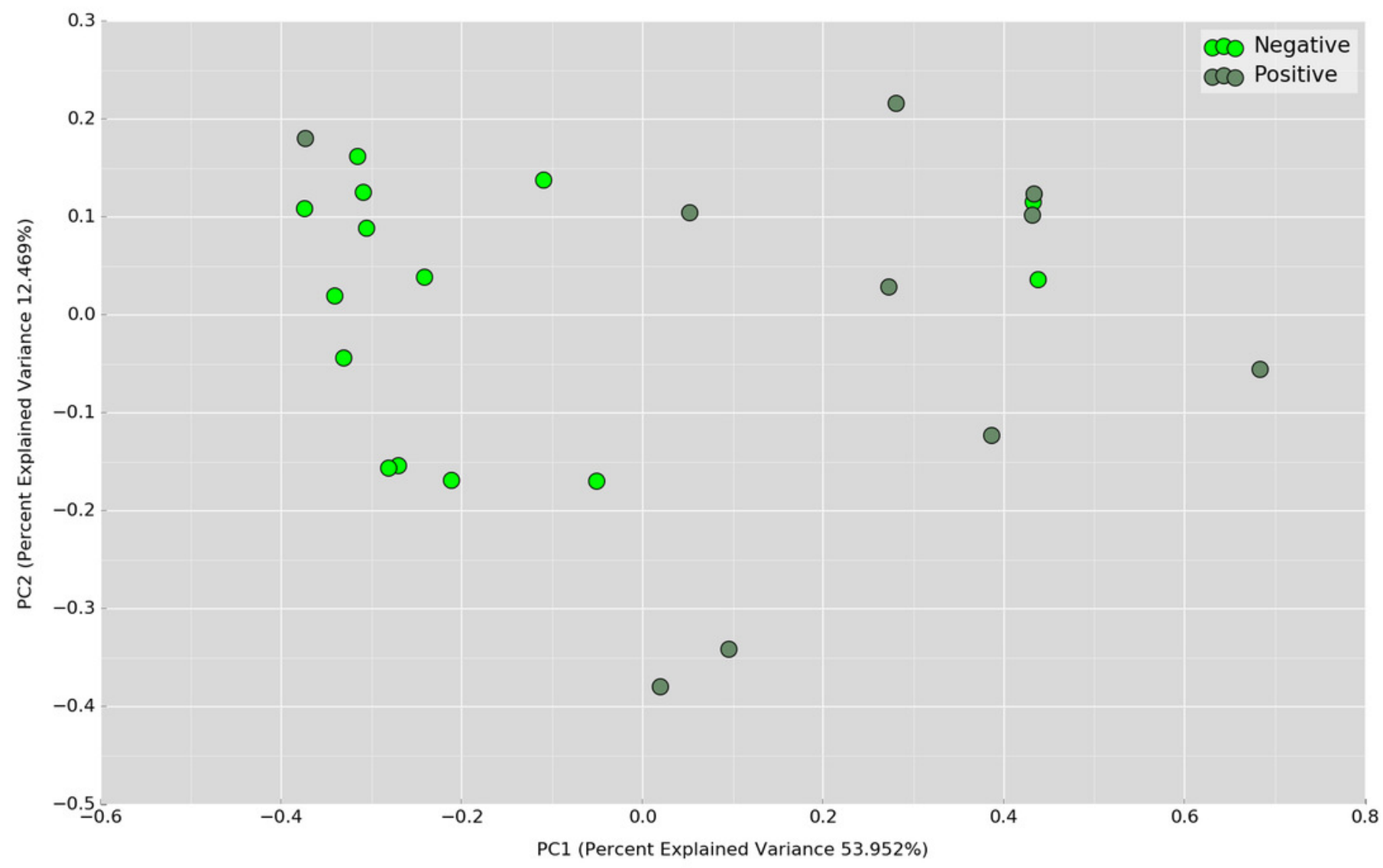




\section{Figure 6}

Identification of bacterial phyla and genera with significant differences between the UBT-negative and UBT-positive daytime samples using LEfSe analysis.

(A) Significantly differentially abundant bacterial phyla identified by LEfSe analysis. (B)

Significantly differentially abundant bacterial genera identified by LEfSe analysis.

A

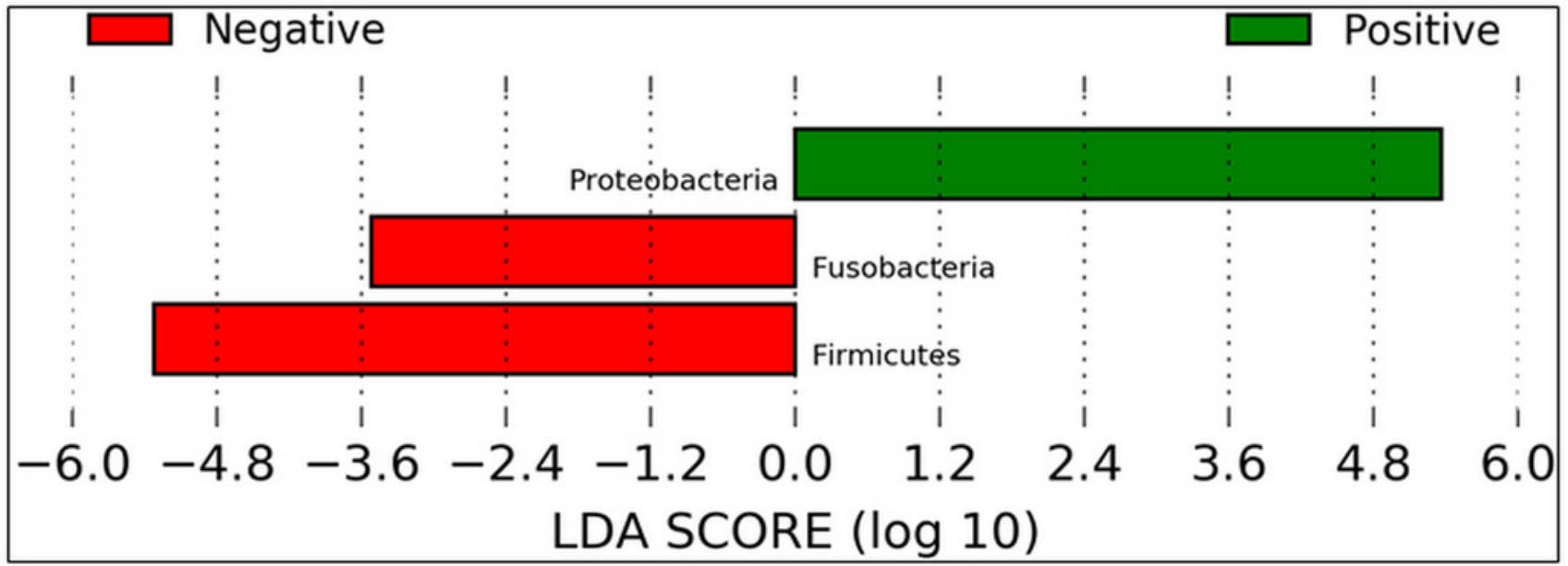

B

\begin{tabular}{|c|c|c|c|c|c|c|c|c|c|c|}
\hline$\square$ & Neg & ative & & & & & & ᄃ & Posi & \\
\hline ! & $!$ & $!$ & $!$ & ! & & $!$ & $!$ & ! & $!$ & ! \\
\hline & & & & Pseudomon & & & & & & \\
\hline & & & & Roseomon & & & & & & \\
\hline & & & & & & usobacțerium & & & & \\
\hline & & & & & & Solobacterium & & & & \\
\hline & $\vdots$ & & & & & taemopḥilus & & & & \\
\hline & & & & & & treptococcus & & & & \\
\hline 1 & 1 & 1 & 1 & 1 & & 1 & 1 & $i$ & $i$ & $i$ \\
\hline-6.0 & -4.8 & -3.6 & -2.4 & -1.2 & 0.0 & 1.2 & 2.4 & 3.6 & 4.8 & 6.0 \\
\hline & & & & LDA SCC & DRE & $=(\log 10$ & & & & \\
\hline
\end{tabular}




\section{Table $\mathbf{1}$ (on next page)}

Demographic characteristics of volunteers recruited in this study. 
1 Table 1. Demographic characteristics of volunteers recruited in this study

2

\begin{tabular}{|l|cc|}
\hline \begin{tabular}{|l} 
Participant characteristics \\
Age
\end{tabular} & UBT-positive $(\mathbf{N}=10)$ & UBT-negative $(\mathbf{N}=14)$ \\
\hline $20-29$ & 4 & 7 \\
\hline $30-39$ & 4 & 6 \\
\hline 40 and above & 2 & 1 \\
\hline Gender & & 9 \\
\hline Male & 5 & 5 \\
\hline Female & 5 & \\
\hline
\end{tabular}

3 